\title{
LOW FREQUENCY LONGITUDINAL RELAXATION OSCILLATION
}

\author{
Yujong Kim*, C. Kim, K. H. Kim, M. H. Chun, J. S. Yang, I. H. Yu \\ M. Kwon, J. Y. Huang, S. H. Nam, M. H. Cho, W. Namkung, and I. S. Ko \\ Pohang Accelerator Laboratory, POSTECH, Pohang 790-784, Korea
}

\begin{abstract}
From the recent data analysis of the longitudinal feedback system and the streak camera, we have found a longitudinal relaxation beam oscillation whose frequency is a multiple of $36 \mathrm{~Hz}$. When the relaxation oscillation is generated, horizontally oscillating wide beams are observed at the diagnostic beamline, and the beam lifetime is fluctuated. The strength of the longitudinal coupled bunch mode instabilities is also modulated sinusoidally. We have described the observed longitudinal relaxation oscillation in the Pohang Light Source storage ring.
\end{abstract}

\section{INTRODUCTION}

In the Pohang Light Source (PLS) storage ring, we have occasionally observed a special longitudinal relaxation oscillation which generates the sinusoidal modulation in the synchrotron oscillation as well as the strength of the coupled bunch mode instabilities (CBMI's). Although our beam bursting behavior is sinusoidal rather than sawtooth, our relaxation oscillation is a kind of the sawtooth instability [1]. When the amplitude of the longitudinal relaxation oscillation is high, horizontally oscillating wide beams are observed at the diagnostic beamline, and the beam lifetime is fluctuated due to the sinusoidal modulation in the synchrotron oscillation. The beam relaxation or bursting is generally generated when the amplitude of the azimuthal quadrupole mode of the CBMI is high enough. However, the bursting is always small when the amplitude of the azimuthal sextupole mode of the CBMI is higher than that of the quadrupole mode. Therefore, characteristics of our longitudinal beam relaxation oscillation is very similar to the new sawtooth instability which is recently observed at the SLAC damping ring after upgrading the vacuum chambers with a smaller inductance [2]. In addition to these, we have found following new properties of the relaxation oscillation at the PLS storage ring: Firstly, the relaxation frequency is always a multiple of $36 \mathrm{~Hz}$, and the strong relaxation oscillation is generated within $200 \mathrm{~Hz}$. Secondly, the beam relaxation can be generated when the temperatures of the RF cavities are not tuned properly though the single bunch beam current is low. Finally, the strongest mode of the quadrupole CBMI's is different from that of the dipole modes when the low frequency relaxation oscillation is strong. We have described the observed properties and a curing method of the longitudinal beam relaxation oscillation in the PLS storage ring by using the streak camera

\footnotetext{
*yjkim@POSTECH.edu
}

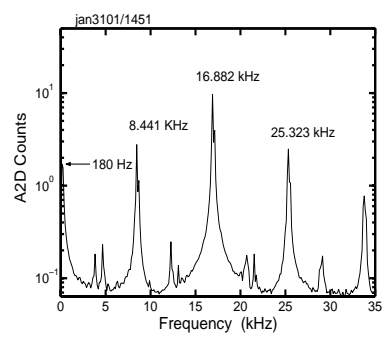

(a)

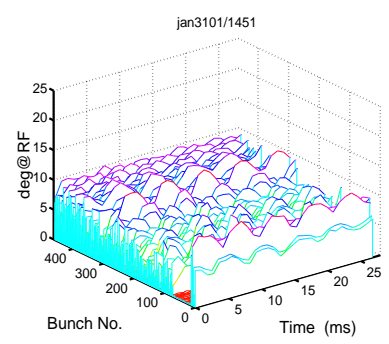

(b)
Figure 1: Beam signal spectrum whose unit is the analogto-digital (A2D) count of the LFS (a) and its time domain 400-bunch synchrotron oscillation amplitudes (b) when the $180 \mathrm{~Hz}$ beam relaxation is generated at $125.97 \mathrm{~mA}$, $2.5 \mathrm{GeV}$. Since we have turned off one of four RF stations at $2.5 \mathrm{GeV}$, the synchrotron or the dipole, the quadrupole, and the sextupole mode frequencies are $8.441 \mathrm{kHz}, 16.882 \mathrm{kHz}$, and $25.323 \mathrm{kHz}$, respectively. Note that the amplitudes of the quadrupole mode and the $180 \mathrm{~Hz}$ relaxation are high.

and the longitudinal feedback system (LFS) as data acquisition systems (DAS).

\section{RELAXATION PROPERTIES}

\subsection{CBMI Modes Vs. Relaxation Amplitude}

When the amplitude of the $180 \mathrm{~Hz}$ beam relaxation is high as shown in Fig. 1(a), the synchrotron oscillation amplitude as well as the strength of the longitudinal CBMI's are modulated sinusoidally with the relaxation frequency as shown in Fig. 1(b). At the same time, we have observed the horizontally oscillating wide beams at the diagnostic beamline due to the relaxation. We have investigated the relation of the beam relaxation amplitude with respect to the amplitude ratio of the quadrupole mode to the sextupole mode of the CBMI. According to our observations, the amplitude of the beam relaxation oscillation is generally high whenever the amplitude of the quadrupole mode of the CBMI or the amplitude ratio of two CBMI modes is high enough as shown in Figs. 1(a) and 2(a). When the strength of the CBMI becomes further higher by increasing the beam current or changing temperatures of the RF cavities, the amplitude of the quadrupole mode is slowed down, and at the same time, that of the sextupole mode becomes higher as shown in Fig. 2(b), In this case, the beam relaxation amplitude within $200 \mathrm{~Hz}$ is very small as shown in Fig. 2(a) and (b). Therefore, the beam relaxation is stopped when the amplitude ratio of two CBMI modes is small. These facts 


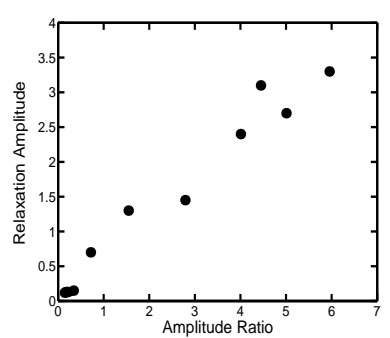

(a)

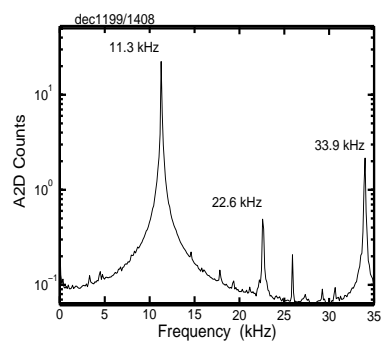

(b)
Figure 2: Beam relaxation amplitude (A2D count) with respect to the amplitude ratio of the quadrupole mode to the sextupole mode of the CBMI (a) and beam signal spectrum (b) when the amplitude ratio of two CBMI modes is small enough at $118.55 \mathrm{~mA}, 2.04 \mathrm{GeV}$. Here, the synchrotron frequency is $11.3 \mathrm{kHz}$ at $2.04 \mathrm{GeV}$.

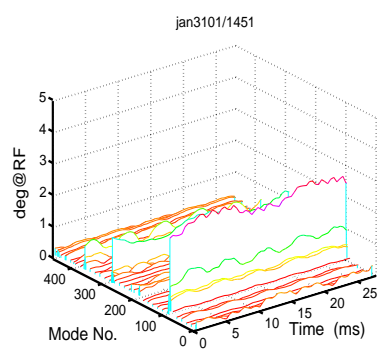

(a)

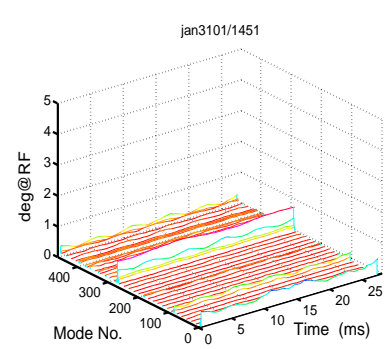

(b)
Figure 3: Time evolution of the quadrupole CBMI's (a) and the dipole CBMI's (b) when the $180 \mathrm{~Hz}$ relaxation oscillation of Fig. 1 is generated.

are well agreed with the new sawtooth instability recently observed at the SLAC damping ring [2].

\subsection{Strongest CBMI Mode}

From recent investigations, we have found that the strongest mode of the quadrupole CBMI's is different from that of dipole modes when a strong relaxation is generated. As one of such cases, the strongest mode of the quadrupole CBMI's is 90 due to $1596.4 \mathrm{MHz}$ higher order mode (HOM) of the RF cavities as shown in Fig. 3(a). On the contrary, the strongest mode of the dipole CBMI's is 279 due to the $1301.1 \mathrm{MHz}$ HOM of the RF cavities as shown in Fig. 3(b). Generally, the strength of the strongest dipole mode is more higher than that of the quadrupole mode. However, in the case of the relaxation oscillation, the strength of the strongest quadrupole mode 90 is about two times higher than that of the strongest dipole mode 279 as shown in Fig. 3. Therefore, the beam oscillation is due to mainly the quadrupole mode instead of the dipole mode when the strong relaxation oscillation is generated as shown in Fig. 4(a) and (b). Here, the longitudinal beam motion is very complex due to the combination of the dipole, the quadrupole, and the sextupole modes, and the trans-

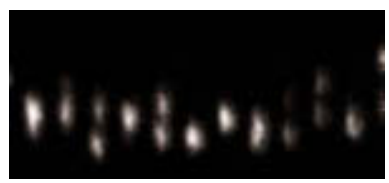

(a)

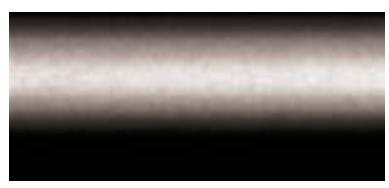

(c)

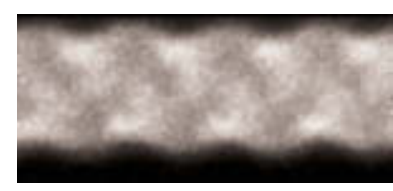

(b)

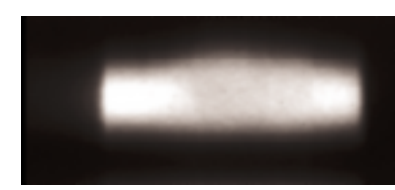

(d)
Figure 4: Dual scan mode streak camera images of 400bunch beam motion when the $36 \mathrm{~Hz}$ relaxation is generated at $200 \mathrm{~mA}, 2.04 \mathrm{GeV}$ (a), when the $180 \mathrm{~Hz}$ relaxation of Fig. 1 is generated (b), when the $180 \mathrm{~Hz}$ relaxation oscillation of Fig. 1 is stopped by the temperature tuning at $121.39 \mathrm{~mA}, 2.5 \mathrm{GeV}$ (c), and when the $36 \mathrm{~Hz}$ relaxation is generated at $165.0 \mathrm{~mA}, 2.5 \mathrm{GeV}$ (d). The maximum time scales of the horizontal axes from (a) to (d) are $100 \mathrm{~ns}$, $500 \mu \mathrm{s}, 500 \mu \mathrm{s}$, and $50 \mathrm{~ms}$, respectively, and those of the vertical axes are 500 ps, 500 ps, 500 ps, and 300 ps, respectively.

verse beam shape is a kind of a dumbbell. Due to the bunch lengthening, the beam lifetime is increased by about five hours than the normal value.

\subsection{Multiple Relaxation Frequency}

The beam relaxation frequency in the PLS storage ring is always a multiple of $36 \mathrm{~Hz}$ according to the beam instability status. Therefore, we have occasionally observed the low frequency beam relaxations such as 36, 72, 108, 144, and $180 \mathrm{~Hz}$ as shown in Fig. 4(d). In this case, the strength of the CBMI is modulated by the relaxation frequency as shown in Fig. 3(a) where the modulation frequencies of one CBMI with the mode number of 90 is $72 \mathrm{~Hz}$ and $540 \mathrm{~Hz}$. However, the other CBMI with the mode number of 89 is much strongly modulated by the $180 \mathrm{~Hz}$ relaxation due to its higher relaxation amplitude. Although the strength of one CBMI with the mode number of 90 is about two times higher than that of 89 , and the former is modulated by $72 \mathrm{~Hz}$ and $540 \mathrm{~Hz}$ relaxations, dominant modulations of all bunches are generated by the $180 \mathrm{~Hz}$ relaxation with the highest relaxation amplitude as shown in Fig. 1. Since the $36 \mathrm{~Hz}$ relaxation frequency is far from the RF noise frequency or the AC ripple frequency, further deep investigation will be needed to understand why the relaxation frequency is always a multiple of $36 \mathrm{~Hz}$.

\section{CURE OF RELAXATION}

Generally, we can shift the HOM frequencies of the RF cavities to avoid any resonance with those of the beam spectra by changing the temperatures of the RF cavities. 


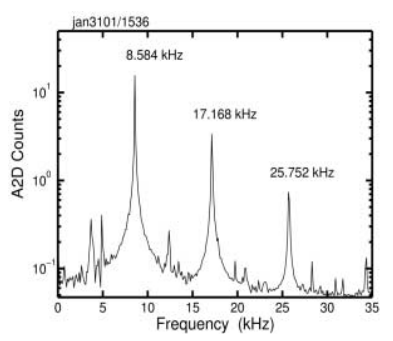

(a)

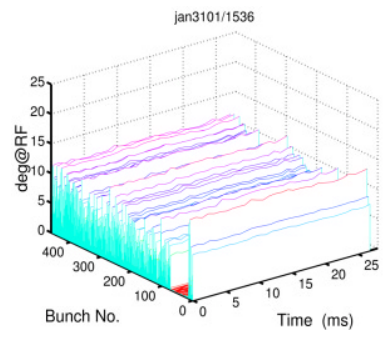

(b)
Figure 5: Beam signal spectrum (a) and its time domain 400-bunch synchrotron oscillation amplitudes (b) when the $180 \mathrm{~Hz}$ beam relaxation oscillation of Fig. 1 is damped by tuning the temperatures of the RF cavities at $121.39 \mathrm{~mA}$, $2.5 \mathrm{GeV}$.

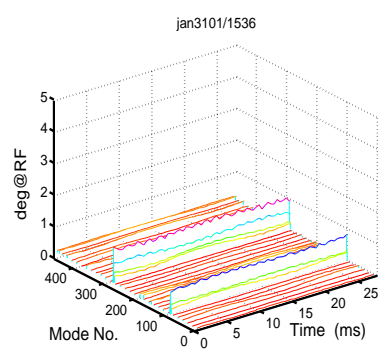

(a)

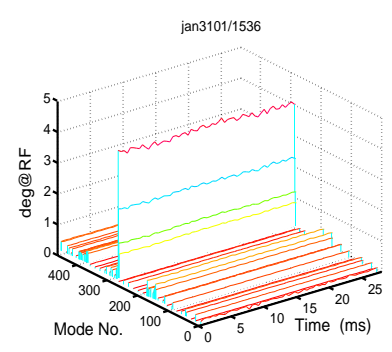

(b)
Figure 6: Time evolution of the quadrupole CBMI's (a) and the dipole CBMI's (b) when the $180 \mathrm{~Hz}$ beam relaxation oscillation of Fig. 1 is damped by tuning the temperatures of the RF cavities at $121.39 \mathrm{~mA}, 2.5 \mathrm{GeV}$.

Therefore, the strength of CBMI's can be controlled by the temperatures tuning [3]. By forcing the amplitude of the quadrupole mode smaller than that of the dipole mode via the temperature tuning, we can convert the relaxation oscillation to the normal CBMI's without any low frequency relaxation [4]. In the PLS storage ring, the $180 \mathrm{~Hz}$ relaxation oscillation of Fig. 1 can be cured by decreasing the temperature of the fourth RF cavity by $1{ }^{\circ} \mathrm{C}$ as shown in Figs. 4(c), 5, and 6. The amplitude of the $180 \mathrm{~Hz}$ relaxation is less than $0.1 \mathrm{~A} 2 \mathrm{D}$ count, and the amplitude of the quadrupole mode is downed while that of the dipole mode is increased as shown in Fig. 5(a). All $180 \mathrm{~Hz}$ modulations in the synchrotron oscillation and the strength of CBMI's are disappeared as shown in Figs. 5(b) and 6. When the relaxation oscillation is stopped by the temperature tuning, the strength of the strongest dipole mode 279 is about four times higher than that of the strongest quadrupole mode 90 as shown in Fig. 6. In this case, the beam oscillation is due to mainly the dipole mode, and the bunch length is reduced as shown in Fig. 4(c). The transverse beam shape is returned to the normal shape of the ellipse, and the beam lifetime is also reduced to the normal value.

According to one simulation model [1], this relaxation oscillation can be explained as following: Under the high intensity or the strong CBMI status, electrons can be randomly excited when the the synchrotron radiation is emitted. In this case, the electrons within a bunch can easily diffuse to other stable fixed point or the tail sub-bunch at the downstream through the unstable fixed point. The electrons within the tail sub-bunch make their own wake and will move to the head sub-bunch at the upstream when they lose their energy due to their own wake field. At the same time, the remaining electrons within the head sub-bunch will move backwards when the electron number is reduced. This means that the RF phase errors of these electrons are reduced to zero. By the help of the radiation damping, two sub-bunches suddenly become one at some point with the nonzero RF phase error when the potential barrier between two sub-bunches is small enough. Since the new single bunch has the over-dense intensity and a large phase error, the new bunch will execute a large amplitude synchrotron oscillation. Therefore, the electrons within the new single bunch will start the diffusion again via the same way as previous processes. By repeating the bunching and then diffusion, the electrons will execute the relaxation oscillation continuously. This model is well agreed with our observation as shown in Fig. 4(a) where we can clearly observe several single bunches with the over-dense intensity and several sub-bunches with the sparse intensity.

\section{SUMMARY}

We have observed the low frequency relaxation oscillation in the PLS storage ring when the strength of quadrupole CBMI is high enough. The continuous bunching and diffusion process due to the over-dense intensity or the strong CBMI may generate the low frequency relaxation oscillation. Although the relaxation has many similarities to the new sawtooth instability, our relaxation frequency is always a multiple of $36 \mathrm{~Hz}$. We can cure the relaxation oscillation by converting the relaxation oscillation to the normal CBMI's without any relaxation via the temperature tuning of the RF cavities.

\section{REFERENCES}

[1] R. Baartman and M. D'Yachkov, in Proc. 1995 Particle Accelerator Conf., Dallas, USA, 1995, pp. 3119-3121.

[2] B. V. Podobedov, SLAC Technical Report No. SLAC-R-543, 1999.

[3] M. Kwon et al., in Proc. 1999 Particle Accelerator Conf., New York, USA, 1999, pp. 899-901.

[4] A. Wrulich et al., in Proc. 5th European Particle Accelerator Conf., Sitges, Spain, 1996, pp. 1108-1110. 